\title{
Management implications of modeling invasion by Allocasuarina huegeliana in kwongan heathland
}

\author{
$\underline{\text { N. Shackelford }}^{\text {a }}$, M. Renton ${ }^{\text {a,b,c }}$, M. P. Perring ${ }^{\text {a }}$ and R. J. Hobbs ${ }^{\text {a }}$ \\ ${ }^{a}$ University of Western Australia, School of Plant Biology, 35 Stirling Highway \\ Crawley WA 6009, Australia \\ ${ }^{b}$ Cooperative Research Centre for National Plant Biosecurity, Australia \\ ${ }^{c}$ Centre of Excellence for Climate Change, Forest and Woodland Health, The University of Western \\ Australia \\ Email: shackn01@student.uwa.edu.au
}

\begin{abstract}
Biotic invasion is a recognized global issue that can lead to biodiversity and ecosystem function loss. It is caused by and interacts with a whole suite of other factors in environmental degradation: climate change, nutrient deposition, disturbance regime shifts, and others. The link between disturbance regime shifts and biotic invasion is particularly well developed in the literature. It is increasingly apparent that in systems where disturbance and invasion are interactively co-occurring, addressing one without addressing the other often leaves both problems not fully resolved. By manipulating the disturbance regime of interest, the disturbance-linked spread of an invasive species can often be controlled. However, the knowledge base necessary for the appropriate use of disturbance as a management tool is difficult to gain from historical, experimental, or observational studies. Therefore, we developed a simulation modeling approach to inform disturbance-based management of invasion.
\end{abstract}

As a case study, we focus on a native invasive tree in the kwongan heathland of Western Australia. We constructed an individual-based population model of its spread into the system from an adjacent seed source. The model was parameterized from empirically-derived data on important life history stages of the tree, including reproduction, dispersal, germination, aging, and death. Because of potential density-dependence in the population spread, we also included stochasticity through the use of a single 'seasonal suitability' parameter that determined seed production, seedling mortality, and initiation of reproductive maturity.

The management strategy of primary concern was fire over a range of intervals and over two interval types: regular fire and a probabilistic randomized regime. We confirmed parameter and model assumption details with local experts and found that outputs of the model were supported by available field data. With an understanding of the modeling technique limitations, we were able to make informative recommendations on the use of fire as a control technique for invasion in the heathland. These techniques could be generalized to assess fire in other systems worldwide, or further to assess the efficacy of any major control effort in population management.

Keywords: individual-based model, sheoak, disturbance, management, fire 


\section{INTRODUCTION}

Biotic invasion is widely recognized as a driver of global change through its direct and indirect effects on local biodiversity and ecosystem functioning. Invasion often occurs in conjunction with a list of other humaninduced causes of global change such as climate change, habitat loss, nitrogen deposition, and disturbance regime shifts. There is growing recognition that these ecological drivers have strongly interactive relationships with biotic invasion (Didham et al., 2007), and that management must attempt to mitigate both the individual and interactive forces in a system. Thus, when managing biotic invasion, the root cause of the invasion must be understood and addressed to fully confront the issue.

The link between biotic invasion and disturbance has been particularly well established in theoretical, observational, and experimental studies (Hobbs \& Huenneke, 1992). The relationship can be strongly interactive, and it is argued that in these cases, the invasion cannot be managed without managing the disturbance as well (Hobbs, 1991). This can be accomplished through utilizing disturbance itself as the management tool. However, this requires knowledge about a system on multiple scales, both spatial and temporal, as disturbance can often have impacts both in the long and short term, and over both large and small scales. Collecting data on multiple scales can often be impractical. Additionally, historical data can be inadequate in drawing strong conclusions about past disturbance regimes. It is often incomplete and relies on anecdotal evidence from sources ranging from untrained travelers to expert scientists. The lack of information can create serious barriers to the use of disturbance as a management strategy. Improperly informed decision-making can potentially have long-term, landscape-scale repercussions.

One method which can help managers overcome major information gaps is simulation modeling. Population modeling techniques are commonly utilized in invasive species management to assess the efficacy of direct control measures (Buckley et al., 2003; Taylor \& Hastings, 2004). These models often span very large spatial and temporal scales and simulate manual and chemical control strategies to predict optimal strategies of application (Higgins \& Richardson, 1996; Parker, 2000; Schreiber \& Lloyd-Smith, 2009). These methods have rarely been used to take into account indirect control methods, but can be expanded to look at the efficacy of disturbance-based management over long time periods across entire systems.

\section{PROJECT SUMMARY}

In this study, we investigated population modeling techniques to assess fire as a disturbance-based management tool for the control of a native invasive in kwongan heathland, a fire-prone Australian system. We focused on Tutanning Nature Reserve, located approximately 35 kilometers from Pingelly, Western Australia. It is a highly diverse reserve with approximately 15 individual heath patches contained in a mosaic of woodland and exposed granite outcrop systems. In the past 30 years, large biodiversity losses have been recorded in eleven of the reserve's kwongan patches (Shackelford, et al., unpublished data). Local land managers believe these losses result from invasion by the native tree Allocasuarina huegeliana. Though found in abundance in adjacent systems, A. huegeliana has previously been absent or rare in heath systems (Bamford, 1995; Main, 1993). It is a fire sensitive species, and managers believe its spread is due to an interaction with local fire regime shifts that have occurred since European colonization (personal communication, C. Yates, 2010) Fire intervals are currently at 50-70 years between fires, and land managers lack full understanding of resulting implications for the invasion process. Sound experimental or observational data is difficult to collect over such a large temporal scale. Therefore in coordination with the Department of Environment and Conservation (DEC), we designed an empirically-based population model to analyze the effects of a range of fire intervals. Our goal was to create a model strongly linked with the specific ecology of the system and determine whether it could be used to reach informative conclusions about disturbance-based management. Because the species is native, eradication in and around these patches is not desirable; rather, containment of its spread into the heath is the final goal.

\section{MODEL STRUCTURE}

\subsection{Overview}

We chose a spatially-explicit individual-based modeling approach. Models of this type allow flexibility in the choice of parameterization and inclusion of stochasticity. The flexibility in parameterization allows the model to be well-supported by available empirical knowledge of a system and the invading species. Though the benefits of the technique are usually overridden by the prohibitive computational power necessary for 
simulations, in a size-limited landscape such as kwongan heathland, we were able to keep computational requirements to a manageable minimum by considering a site approximately 18 ha in total invasible area.

The simulation represents a single heath patch using a grid of square cells. Each cell is approximately $5 \mathrm{~m}^{2}$, with cell size determined by the carrying capacity of 2,000 trees/ha found for a related Casuarinaceae species (Ladd, 1989). Cells have three possible states: absence of A. huegeliana, presence of A. huegeliana seedling, or presence of $A$. huegeliana adult individual.

\subsection{Parameterization}

Many heath patches are located adjacent to an A. huegeliana stand. We therefore include in the model approximately 2 ha of constant seed source located at the bottom end of the simulated site that contains 100 individuals at the initiation of each run. From that persistent seed source the population spreads into the simulated heath patch. Model parameterization is based on existing knowledge of the life history of $A$. huegeliana in heathland. Time steps are annual and include five stages: reproduction by seed-producing adult females, dispersal of seed, germination, aging of existing individuals, and seedling and adult mortality. The model is coded in the $\mathrm{R}$ programming language and is available upon request to the authors. See Table 1 for a complete list of parameter values (adapted from Shackelford, et al., unpublished data).

Table 1: Parameters used in the model and their values

\begin{tabular}{|c|l|c|}
\hline Parameter & \multicolumn{1}{|c|}{ Definition } & Value \\
\hline$F$ & Female probability & 0.4 \\
\hline$R$ & Minimum reproductive age & 9 \\
\hline$X_{0}$ & Cauchy parameter: location & 0 \\
\hline$\Gamma$ & Cauchy parameter: scale & 0.05 \\
\hline$D$ & Adult mortality & $\mu=0.5, \sigma=0.1$ \\
\hline & \multicolumn{1}{|c|}{ Stochastic Parameters } & $\mu=0.82, \sigma=0.06$ \\
\hline$y_{t}$ & Seasonal suitability & $\mu=0.97, \sigma=0.01$ \\
\hline$d_{t}$ & Seedling mortality & $\mu=150, \sigma=30$ \\
\hline$d f_{t}$ & Seedling mortality: post-fire & 1 \\
\hline$s_{t}$ & Viable seed production per tree & 0.9 \\
\hline \multicolumn{2}{|c|}{ Management Parameters } \\
\hline$D f$ & Adult mortality: fire & Adult mortality: managed removal \\
\hline$D r$ & \multicolumn{2}{|c|}{}
\end{tabular}

\section{Reproduction}

Allocasuarina huegeliana is a dioecious species. However, little data exists on exact population proportions between males and females. Maher (2007) performed surveys on 11 of the Tutanning heath patches and recorded $20 \%$ of the total population as fruiting females. Since it was impossible to determine the gender of plants not yet of reproductive maturity in the field, we assumed only $50 \%$ of the population had reached a stage of maturity enabling accurate identification. Based on the determination that $20 \%$ of reproductive adults were female, and assuming the same proportion for non-reproductive juveniles, $40 \%$ of the total population is estimated to be seed-producing female. This variable is parameterized in the model as $F=0.4$, the probability that newly produced seed is female. The nominal age at which females begin producing seed has been found to be approximately nine years. This parameter, $R$, is subject to some stochasticity, as detailed further below in Section 3.3.

For female trees of reproductive maturity, effective seed production is defined within the model as the number of seeds that are produced, are viable, and find suitable microenvironments for germination. For computational simplicity, we estimate this process as a single parameter, viable seed production $s_{t}$. This 
parameter was difficult to base in empirical study. Therefore we calibrated the parameter by running the simulation with a range of values and adjusting the value so that model results correlated with existing density data collected in the field. The parameter changes stochastically between years. However, all reproductive trees have equal $s_{t}$ within a timestep.

\section{Dispersal}

Once individual trees have produced seeds, they are dispersed across the landscape according to a Cauchy distribution:

$$
\left.f\left(x ; x_{0}, \gamma\right)=\gamma / \pi^{*}\left(x-x_{0}\right)^{2}+\gamma^{2}\right]
$$

with location $x_{0}=0$ and scale $\gamma=1.25$. This simulates a seed rain in which $98 \%$ of the seed falls within 20 cells, or 45 meters, of the parent tree, which matches empirically based estimates (Standish et al., 2007). The Cauchy distribution is a leptokurtic distribution that allows long distance dispersal events. Dispersal kernels accounting for long distance events have been found to represent seed dispersal relatively well for winddispersed tree species such as A. huegeliana in many instances (Howe \& Smallwood, 1982). Once dispersal distance for each individual seed is determined, the direction of dispersal is randomly chosen from a uniform distribution ranging from 0 to $2 \pi$. In the case of a negative dispersal distance, dispersal occurred in the opposite direction of that produced by the uniform distribution selection. Because of the nature of kwongan patch size limitation, we did not simulate a 'wrap-around' function for seeds dispersing beyond the site; if seeds are dispersed off the edges of the simulation, they are considered to be lost.

\section{Germination}

Though cell size accommodates more than a single seedling, field observations have been that intra-specific competition leads to a single individual per area depicted by each cell. Therefore, if the cell in which a seed is distributed is currently occupied, seed mortality is assumed. Otherwise, once distributed across the landscape, all seeds are assumed to germinate. Because mortality occurs after germination in the chronology of a single timestep, germinated seeds are designated to have an age of 1 . This ensures that seedling mortality, described in detail below, lasts only for two total timesteps of the individual's lifespan, at which point the seedling is assumed to have reached a size that provides resilience to environmental threats such as grazing and drought.

\section{Aging}

All existing individuals except newly germinated seeds increase in age by one year per timestep.

\section{Mortality}

There are two life stages - seedling and adult - and three whole site conditions - unburned, burned, or burned within the last three years. If the site is burned in a timestep, $100 \%$ mortality is assumed for seedlings and adults in the burned heath within that timestep (personal communication, C. Yates, 2010). Though fires occur in generally patchy patterns that would not produce such uniform impacts, we are assuming a managed burn at the high temperatures necessary for maximum A. huegeliana mortality. Under all other conditions, adults are assumed to be capable of surviving environmental stresses and so are designated a set $5 \%$ annual mortality rate to account for natural senescence.

We altered seedling mortality rates dependent upon whether a site has been burned in the last three years or not. Studies on A. huegeliana seedling survival in heath patches were conducted by Maher (2007) in Tutanning Nature Reserve and very low seedling survival rates were found. Due to environmental stress including preferential grazing by native herbivores, an average seedling mortality rate of $82 \%$ was found in vegetated areas. Additionally, Yates et al. (2003) tracked mortality of A. huegeliana over 12 years. The study found no new seedlings over the 12 year span and found that only $13.7 \%$ of the original seedlings had survived; i.e. they experienced an approximately $86 \%$ mortality rate. We therefore set the average seedling mortality under unburned conditions at $82 \%$. This parameter is stochastic and varies annually. If recently burned, we set a higher mortality rate, because recently burned heath sites are generally bare of shrubs and other potential 'nursing' cover that otherwise protects A. huegeliana seedlings. In the same study previously mentioned, seedling mortality found by Maher in bare patches was $97 \%$. Accordingly, for the first three timesteps after a fire event, any seedlings present in the model experienced higher mortality averaging $97 \%$ but again subject to annual stochasticity. Under all conditions, if a seedling survived its first two years, it is assumed to become an 'adult' and is no longer subjected to higher mortality rates due to environmental stress. 


\subsection{Stochasticity}

All environments experience fluctuations in the suitability of climate and environmental conditions for the survival or performance of particular species. That stochasticity is potentially important in population spread and we therefore included three parameters that varied stochastically: seedling mortality, seed production, and age at which a female tree first reproduces. Annual values for seedling mortality and viable seed production and the probability of female trees starting to produce seeds all depend on seasonal factors such as rainfall, so are likely to be tightly correlated. Therefore, in the model, all three depend upon a single parameter that we termed the 'seasonal suitability' parameter, $y_{t}$. Seasonal suitability ranges from 0 to 1 and is randomly chosen at the beginning of each timestep from a truncated normal distribution with mean $\mu=0.5$ and standard deviation $\sigma=0.1$. Seedling mortality is calculated from a normal distribution function varying from 0 to 1 , using $y_{t}$ as the input quantile. This is then truncated at 0 and 1 . The parameters for this normal distribution are $\mu=0.97$ and $\sigma=0.01$ for the three years immediately post-fire and $\mu=0.82$ and $\sigma=0.06$ for all other years. Variance was set so that in a 'worst' year mortality was approximately 0.99 . Viable seed production is similarly calculated using $y_{t}$ from a normal distribution with $\mu=150$ and $\sigma=30$. For example, if $y_{t}=0.5$ for a given year, representing a median season, then seedling mortality and viable seed production for that year will both be at their mean value. If seasonal suitability is higher than 0.5 (a 'good year'), seedling mortality will be less than average and seed production greater than its average. The final stochastic parameter, the age at which a female tree first reproduces, is determined by seasonal suitability only insofar as it is a limiting factor on seed production initiation. Once an individual female is older than the nominal reproductive age of 9 years, it will begin producing seed in the first timestep in which $y_{t} \geq 0.5$. Once seed production has been initiated, it continues every timestep until mortality.

\subsection{Sensitivity Analysis}

For good prediction of population behavior it is essential to accurately parameterize the model, but some parameters are likely to affect outcomes more than others. This can be addressed through parameter sensitivity analysis. Though we had empirical data supporting most of our parameters, there is always concern as to the level of impact a single, uncertain parameter might have on the outcome of the simulation. For all sensitivity analyses, we tested two scenarios of a regular 30 year fire interval over 2,000 timesteps and a regular 65 year fire interval over 2,000 timesteps. We first assessed the impact of reduced post-fire mortality, decreasing the probability of mortality for individuals in a fire from $100 \%$ to $50 \%$ in $10 \%$ increments. We assessed both the parameter values of the Cauchy kernel and the dispersal kernel itself. Parameter values for the Cauchy kernel were tested at $+/-50 \%$ of the model values, altering the average distance seeds fall from the parent tree. However, the choice of kernel has been shown to dramatically affect patterns of spread and invasion (Kot et al., 1996). As a contrast to the heavily fat-tailed Cauchy kernel, we tested the Weibull distribution and the lognormal distribution, two leptokurtic kernels that are less weighted away from the mean. We parameterized them to have $98 \%$ of the seed fall within the same $45 \mathrm{~m}$ distance as used for the Cauchy distribution definition. Finally, we tested the percentage of reproductive females within the population and the calibrated parameter $s_{t}$, viable seed production per reproducing female tree. Similar to the dispersal parameters, we tested the proportion of reproductive females at $+/-50 \%$ the model parameter values. Because seed production is a normal distribution that varies stochastically, we altered the average value $+/-50 \%$. Each parameter that was tested in the analysis was tested individually, and not in combination with other parameters.

\subsection{Management Strategies}

We investigated fire occurring in set, regular frequencies ranging from 10-80 years between fires in 5 year increments. Additionally, we investigated randomized fire occurrence, with fire frequency determined by annual probabilities corresponding to the same 10-80 frequencies as in the regular simulation; i.e. there was a $10 \%$ chance of fire for each individual timestep in the 10 year randomized regime, a $5 \%$ chance in the 20 year randomized regime, and so on.

\section{RESULTS AND DISCUSSION}

We wanted to assess the adequacy of the model in regards to its empirical soundness. Though most of the parameters were well-grounded in literature, the literature itself is fairly limited. We had local land managers check inputs, assumptions, and outputs and confirm that it seemed well within field evidence (C. Yates, K. Brooks, B. Beecham, personal communication, 2010). The sensitivity analysis showed that the two factors with the most influence over model results were the dispersal kernel and post-fire mortality rates. The dispersal kernel chosen - the Cauchy kernel - is one commonly used in long distance dispersal. Additionally, 
when studying the sensitivity outputs, the Cauchy kernel resulted in population behavior closest to that seen in the field (Figure 1). Post-fire mortality is a parameter potentially under the control of managers, and thus the model's sensitivity to it is not of great concern. The inherent factor untested in our model is the influence of the many environmental variables omitted through our choices in ecological simplification.
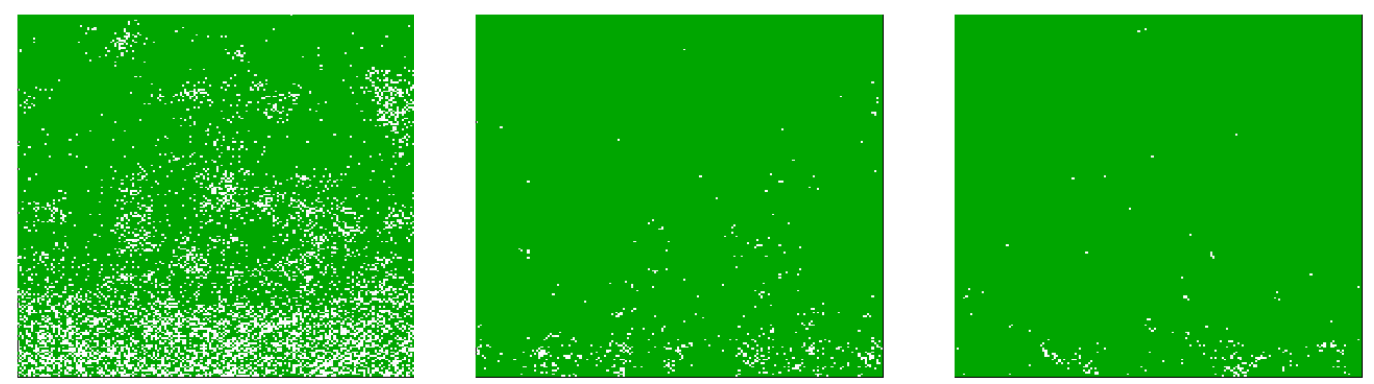

Figure 1: Model output after 65 years without fire using Cauchy distribution (left), Weibull distribution (middle), and the logNormal distribution (right) for seed dispersal. The images are 'aerial' views of the simulated heath space, with green being uninvaded heath and white invaded. In field results, invasion is up to 1425 trees/ha after 70 years without fire, correlating most closely with the Cauchy distribution results.

Independent quantitative model validation was not possible due to our use of the available data to calibrate parameters. Even after calibration, the clear pattern of fire-dependent increase found in the output is not reflective of the more scattered density levels found in the field (Figure 2). However, the data does confirm the significant, positive relationship between time since last fire and density levels. Those sites that contribute most to weakening that relationship are of a substrate types that is thought to be inhospitable to $A$. huegeliana. Though $A$. huegeliana density was found to have a significant relationship with several other environmental variables

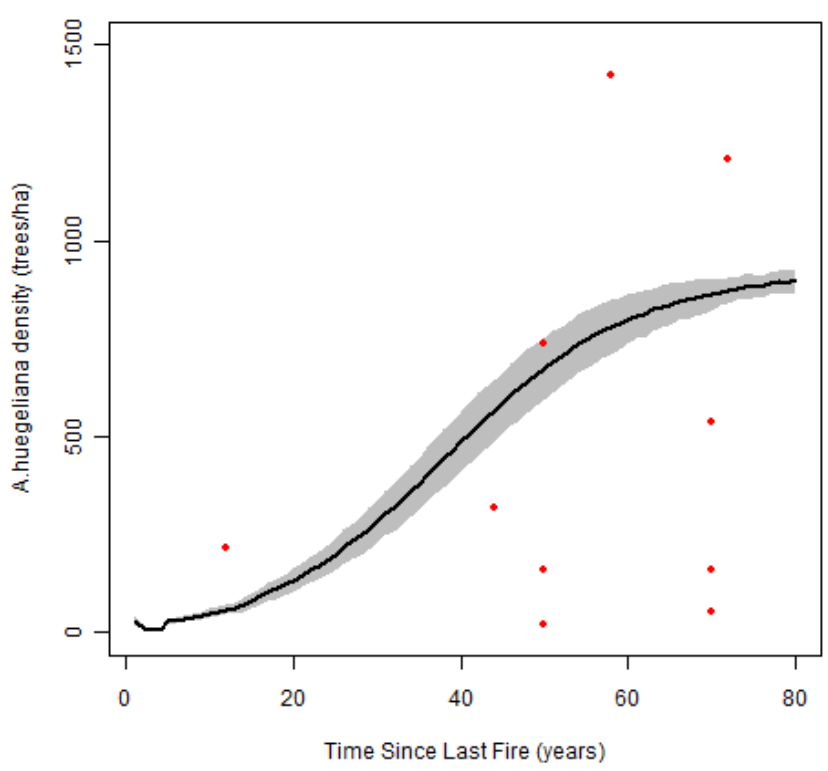

Figure 2: Model density output and real data. The black line represents average $A$. huegeliana densities based on time since last fire as predicted by the simulation model; the surrounding grey is the standard deviation; the red points are field densities at eleven separate sites in Tutanning Nature Reserve (Shackelford, et al., unpublished data) as well, its relationship with fire is the one of primary interest when using disturbance as a management tool. The positive relationship in the data supports the hypothesis that key management strategies can be determined from model results and our model explores the mechanisms behind that relationship, allowing further options to be investigated such as fire paired with managed removal.

Keith and Bradstock (1994) found that a fire regime of alternating short and long intervals would likely be ideal in maintaining the highest levels of diversity in the heathland. This type of study can be paired with our model output to move towards a better understanding of fire management that may address many issues in the system beyond only invasion. Fire as natural disturbance is prominent in many systems worldwide, for example the fireprone South African chaparral and the North American prairie. This type of modeling can easily be adapted to these other systems in which there is a species or suite of species with fire-dependent population behavior. 
Empirically-based population modeling techniques of the kind used in this study can be expanded to explicitly look at any number of single or combined disturbance-based management strategies. There is enormous variety in management practices that are pivotal in the maintenance of specific species populations. For instance, in a further application of our model, we plan to combine fire with mechanical removal and assess the difference in control efficacy. Similarly, any control method of interest can be included and parameterized to predict management outcomes. Considerations in these applications would be the scale of the model and the nature of the species of concern. Individual-based models are most applicable in instances of relatively low-density species on relatively small scales. High density species like grasses and larger landscape level scales might be better suited for an alternative method of population modeling. Nevertheless, our study showed the applicability of individual-based population modeling in population management under strategic, disturbance-based control measures.

\section{ACKNOWLEDGMENTS}

We would like to thank the Department of Environment and Conservation, specifically Dr. Kristine Brooks[and[Dr. Brett Beecham for their advice and willingness to collaborate. In addition, we must acknowledge the support and guidance of the ERIE Research Group and its individual members.

\section{REFERENCES}

Bamford, M. (1995). Exploring Wheatbelt Woodlands. Perth: Department of Conservation and Land Management.

Buckley, Y. M., Briese, D. T., \& Rees, M. (2003). Demography and management of the invasive plant species Hypericum perforatum. II. Construction and use of an individual-based model to predict population dynamics and the effects of management strategies. Journal of Applied Ecology, 40(3), 494-507.

Didham, R. K., Tylianakis, J. M., Gemmell, N. J., Rand, T. A., \& Ewers, R. M. (2007). Interactive effects of habitat modification and species invasion on native species decline. Trends in Ecology \& Evolution, 22(9), 489-496.

Higgins, S. I., \& Richardson, D. M. (1996). A review of models of alien plant spread. Ecological Modelling, 87(1-3), 249-265.

Hobbs, R. (1991). Disturbance a precursor to weed invasion in native vegetation. Plant Protection Quarterly, 6(3), 99-104.

Hobbs, R. J., \& Huenneke, L. F. (1992). Disturbance, Diversity, and Invasion - Implications for Conservation. Conservation Biology, 6(3), 324-337.

Howe, H. F., \& Smallwood, J. (1982). Ecology of seed dispersal. Annual review of ecology and systematics, $13,201-228$.

Keith, D. A., \& Bradstock, R. A. (1994). Fire and Competition in Australian Heath - a Conceptual-Model and Field Investigations. Journal of Vegetation Science, 5(3), 347-354.

Kot, M., Lewis, M. A., \& van den Driessche, P. (1996). Dispersal data and the spread of invading organisms. Ecology, 77(7), 2027-2042.

Ladd, P. G. (1989, 9-11 May 1988). The status of Casuarinaceae in Australian forests. Paper presented at the First National Conference on Australian Forest History, Canberra.

Maher, K. (2007). Encroachment of sandpland heathland (kwongan) by Allocasuarina huegeliana in the Western Australian wheatbelt: the role of herbivores, fire and other factors. Doctor of Philosophy, Murdoch University, Perth.

Main, A. R. (1993). Landscape Reintegration: Problem Definition. In R. J. Hobbs \& D. A. Saunders (Eds.), Reintegrating Fragmented Ecosystems. New York: Springer-Verlag.

Parker, I. M. (2000). Invasion dynamics of Cytisus scoparius: A matrix model approach. Ecological Applications, 10(3), 726-743.

Schreiber, S. J., \& Lloyd-Smith, J. O. (2009). Invasion dynamics in spatially heterogeneous environments. American Naturalist, 174(4), 490-505. doi: 10.1086/605405

Standish, R. J., Cramer, V. A., Wild, S. L., \& Hobbs, R. J. (2007). Seed dispersal and recruitment limitation are barriers to native recolonization of old-fields in western Australia. Journal of Applied Ecology, 44(2), 435-445. doi: DOI 10.1111/j.1365-2664.2006.01262.x

Taylor, C. M., \& Hastings, A. (2004). Finding optimal control strategies for invasive species: a densitystructured model for Spartina alterniflora. Journal of Applied Ecology, 41, 1049-1057.

Yates, C. J., Hopper, S. D., Brown, A., \& van Leeuwen, S. (2003). Impact of two wildfires on endemic granite outcrop vegetation in Western Australia. Journal of Vegetation Science, 14(2), 185-194. 